\title{
A Visualization Environment for Supercomputing-based Applications in Computational Mechanics
}

\author{
Constantine J. Pavlakos ${ }^{1}$ (presenting author)
}

Larry A. Schoof ${ }^{2}$

John F. Mareda ${ }^{3}$

Applied Visualization Group

Sandia National Laboratories

Dept 1425

P. O. Box 5800

Albuquerque, NM 87185

\section{DISCLAIMER}

\begin{abstract}
This report was prepared as an account of work sponsored by an agency of the United States Government. Neither the United States Government nor any agency thereof, nor any of their employees, makes any warranty, express or implied, or assumes any legal liability or responsibility for the ascuracy, completeness, or usefulness of any information, apparatus, product, or process disclosed, or represents that its use would not infringe privately owned rights. Reference herein to any specific commercial product, process, or service by trade name, trademark, manufacturer, or otherwise does not necessarily constitute or imply its endorsement, recommendation, or favoring by the United States Government or any agency thereof. The views and opinions of authors expressed herein do not necessarily state or reflect those of the United States Government or any agency thereof.
\end{abstract}

1. cjpavla@sandia.gov, (505) 845-8550, FAX (505) 844-9297

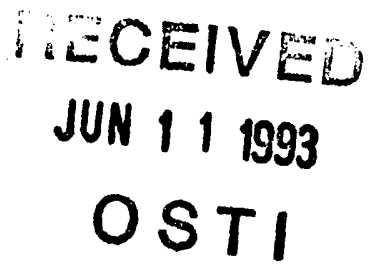

2. laschoo@somnet.sandia.gov, (505) 844-5156, FAX (505) 844-9297

3. jfmared@cs.sandia.gov, (505) 845-8550, FAX (505) 844-9297 


\begin{abstract}
In this paper, we characterize a visualization environment that has been designed and prototyped for a large community of scientists and engineers, with an emphasis in supercomputing-based computational mechanics. The proposed environment makes use of a visualization server concept to provide effective, interactive visualization to the user's desktop. Benefits of using the visualization server approach are discussed. Some thoughts regarding desirable features for visualization server hardware architectures are also addressed. A brief discussion of the software environment is included. The paper concludes by summarizing certain observations which we have made regarding the implementation of such visualization environments.
\end{abstract}

\title{
1.0 Background
}

As a multiprogram engineering laboratory, Sandia National Laboratories (SNL) has major research and development responsibilities for nuclear weapons, arms control, energy, environment, and other areas of strategic importance to national security. To accomplish this diversified mission, analysts employ computational mechanics methods to support the entire laboratory in solving problems in fluid dynamics (steady state and transient, compressible and incompressible), thermodynamics (heat transfer), hydrodynamics (impact physics, penetration mechanics), solid mechanics (structural/ thermal analysis, fracture mechanics, ground subsidence, inpact modeling) and structural dynamics. To assist these analysts in performing these analyses most efficiently, the Applied Visualization Group was formed and tasked to develop a next-generation scientific visualization environment.

For the creation and implementation of this environment, an extensive list of functional specifications was developed in response to users' requests. Included in these specifications were the following requirements/constraints:

- Service 150 scientists / engineers.

- Support 20 simultaneous visualization users.

- Allow efficient visualization of large databases. Analyses currently executed on our supercomputer routinely generate results files between $100 \mathrm{MB}$ and $1 \mathrm{~GB}$ in size. Results files in the 10GB-100GB range are anticipated.

- Allow for visualization at the desktop. Analysts desire to visualize results in their offices where the information used to perform the analysis (e.g. material properties, input loads, model constraints, etc.) is readily available.

- Utilize existing desktop hardware. All users had a display device (workstation or X-terminal) on theil desks but the only commonality was that they were X11 windows devices. A related constraint was to be able to replace desktop displays in the future without requiring the replacement of the entire visualization environment. 
A few motivating factors which drove the users to request the development of a new environment are worthy of note. First, computing resources (i.e., cpu speeds, internal memory sizes, disk sizes, etc.) have matured in the past few years to the point that 3D analyses are now run routinely, although visualization of the results is still quite painstaking. Secondly, users are demanding to interact with their data sets rather than post-process their results using batch-oriented procedures. Previously, "batch graphics" on a supercomputer was the only practical option for some problems due to the size of individual data fields (e.g. a scalar field of temperature at each grid point for one instant in time) as well as the size of the entire results file (10-40 scalar values at each grid point for all time steps) which was unwieldy to download. A third factor, related to the previous two, is the expectation of a consistent response time in visualizing analysis results. Users want to see an image generated at their desk within a certain time threshold, regardless of the complexity of the problenı. Although an acceptable response time has been difficult to quantify, the general consensus of our users is that a few seconds $(<10)$ is satisfactory for generating a complex image.

In order to transform user requirements into a proposed hardware / software solution, it was necessary to understand the application environment.

\subsection{Application Environment}

The analysis environment employed by our engineers / scientists can be divided into three primary phases: problem definition, typically performed on a local server; simulation, executed on a central supercomputer; and visualization, currently done on either a local server or a central supercomputer, depending on the size of the problem. It is critical to understand each of these phases in order to design an environment that allows efficient use of resources. In examining these processes, particularly the visualization process, we determined that database accessibility was a major issue. Indeed, from a high-level perspective, the database is the central hub of the environment and each process is simply an application accessing the hub (see Figure 1). Database issues will be discussed further in Section 6.0, Software Environment.

A required function incorporated into this model is simulation tracking: that is, monitoring the results of an analysis as it is executing. A future evolution of this functionality will be simulation steering, ir which parameters within the simulation are modified when there is evidence that the results are going awry. An Example of this is adaptive meshing, in which the simulation geometry is discretized more finely in regions where the gradient of a calculated variable is very high. 


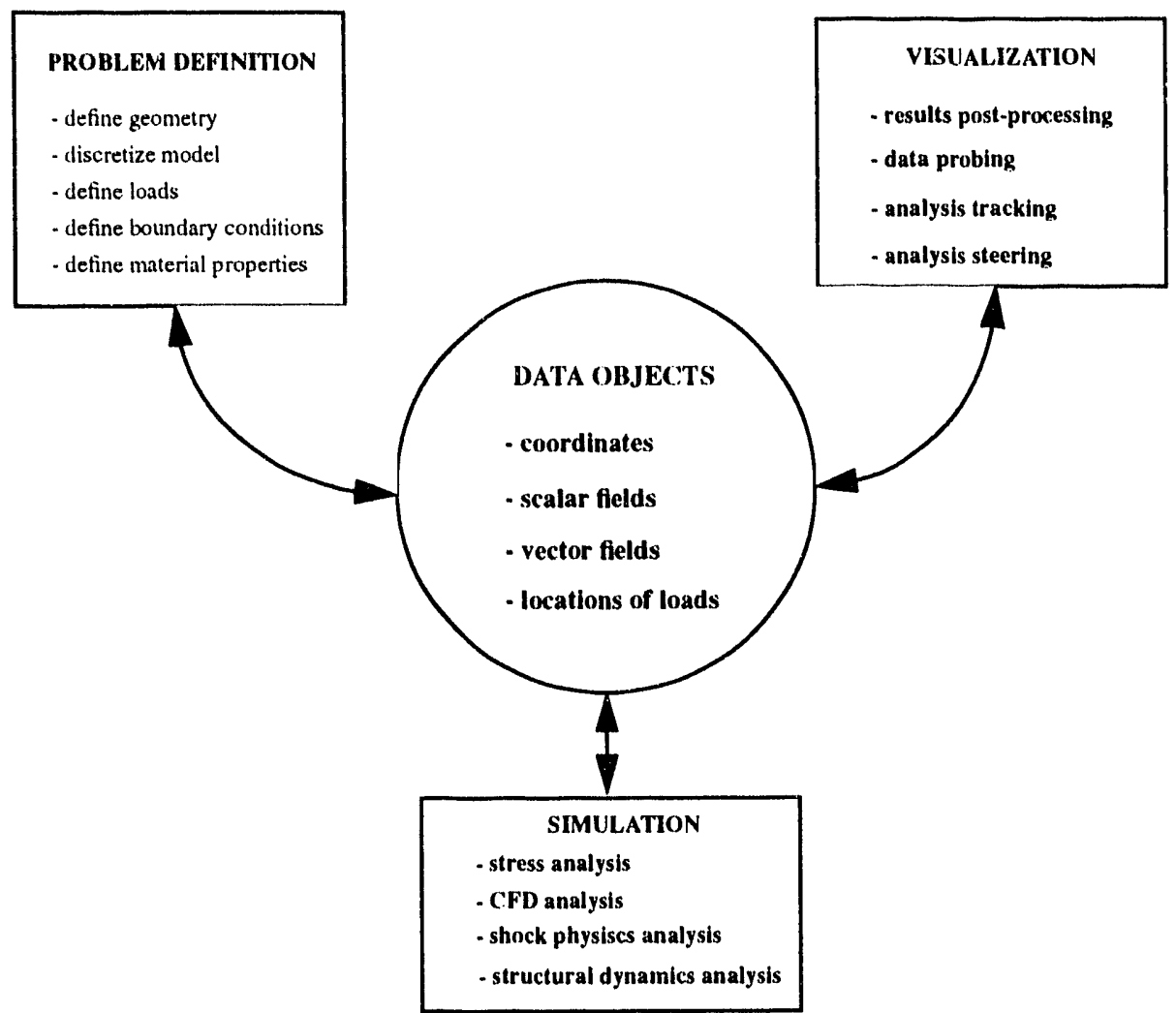

Within this model, the visualization process can be divided into the following four steps, as Upson, et al [1] described: (1) input; (2) filtering into another form that is more informative and perhaps less voluminous; (3) mapping data into geometry; (4) rendering geometry into images. It is important to note that this process involves much more than just rendering objects. Although geometric transformations of an object (the most used function of the rendering step) are important to analysts, the other steps often dominate the entire process, especially when processing large data sets. We have experienced this during our prototyping. In one specific case, a low cost workstation with a fast cpu and no graphics acceleration processed data to produce an image an order of magnitude more quickly than a high end workstation with graphics acceleration, but a slower cpu.

This phenomenon is particularly true with time dependent data sets, in which the analyst is concerned about changes in the time domain, not just the space domain (see pg. 13, Section 6.0, Software Environment). Yet, most special purpose hardware accelerates only the rendering step, leaving the other steps to be performed by a general purpose cpu. What is needed is a balanced approach: maximum processing power available to each of the stages as required. We must be cautious that the complete visualization process is not obscured by the polygons/sec wars being waged among workstation vendors. 


\subsection{Proposed Network / Hardware Configuration}

In order to do highly interactive visualization of supercomputing results, a very tight, high-speed integration is required between the supercomputer(s), large storage facilities, and the visualization components. The goal is to implement the equivalent of a super graphics workstation.

Consider the architecture of a standalone high performance graphics workstation. Such a system typically includes one or more general purpose cpus for computation, a large high-speed disk farm, and extra processing power for pseudo realtime graphics, all bundled together in a tightly integrated system. This architecture is analogous to the proposed network architecture for supercomputing-based applications depicted in Figure 2, which uses a "supernet" to integrate the supercomputing, storage, and visualization components. Based on current technology, alternatives for such a "supernet", such as HiPPI and UltraNet, provide transfer rates on the order of $100 \mathrm{MB} / \mathrm{sec}$ (peak).

FIGURE 2. Proposed Network Architecture

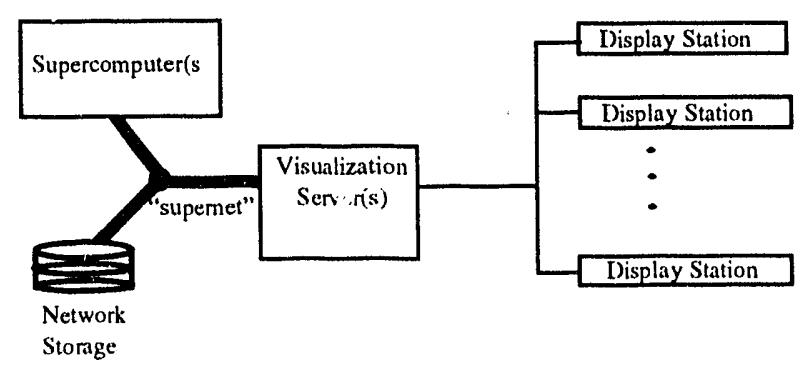

There are a number of alternatives for what kinds of visualization systems to implement and where to put them. Certainly, one alternative is to purchase a high-performance graphics workstation for each user. This, unfortunately, would be prohibitively expensive, since our visualization requirements mandate high-end workstation configurations and we have many users to support.

A second alternative is to provide some smaller number of high-performance graphics workstations at appropriately distributed locations which could be shared. This approach has been attempted several times at Sandia, generally fauling due to users' unwillingness to go down the hall to use a special system -- users prefer to use the system at their desk, even at significantly reduced performance. This approach also has the problem of contention for a physical resource (i.e. the limited number of high-end graphics workstations). 
An additional problem with both of the above alternatives is the implementation of the tight communications interface (i.e. "supernet") with the supercomputer(s) and file store. The number of visualization systems together with their distributed locations would make high-speed communications ver $f$ expensive as well as technically challenging.

This course of reasoning led us to conclude that what we need is a "visualization server" which would provide the aggregate equivalent of some number of high-end graphics workstations, together with the ability to deliver images to desktop displays all acceptable rates.

Conceptually, the capabilities desired for such a visualization server are characterized as follows:

- The server should have the aggregate power of multiple (in our case, preferably 10-20) high performance graphics systems bundled into a single system, accessible by multiple simultaneous users.

- The server should be able to compute images at the same rate as a standalone, high performance graphics system. All that remains is to get the images to the desktop. The difference in graphics performance between using a central visualization server and sitting directly in front of a high-performance graphics workstation should be only the time to transmit the image (not the time to compute it).

- The server should be able to drive conventional desk-top displays, via conventional communications media, as fast as possible.

The concept of a shared, centralized visualization server is not entirely a novel idea. Alliant Computer Systems tried to sell the idea for a long time [2], and others have promoted the concept as well [3]. A number of vendors are currently promoting systems as visualization servers, including Convex and IBM (Power Visualization System). However, no vendor that we are aware of currently meets all of the desired features as we have characterized them.

Some ideas and/or emerging technologies which have the potential to make visualization servers more viable include:

- The concept of generating images, at high performance graphics rates, not just to a local console, but into more general purpose memory, from where the image can be redirected to an arbitrary display (Stellar's Virtual Pixel Map) architecture was a siep in this direction [4]).

- Higher speed networking technology, resulting in higher speed transmission of images to the desktop.

- Various image transmission techniques, including image compression schemes and digital video.

- PEX [5] as a mechanism for distributing the rendering process between the server and the desktop).

Benefits of a centralized visualization server include:

A Visualization Environment for Supercomputing-based Applications in Computational Mechanics 
- Cost-effectiveness. Consider the case of serving 100 users, of which 10-20 need to do vista ization simultaneously.

The cost of purchasing 100 medium-level graphics workstations at $\$ 50,000$ each would be $\$ 5,000,(000$. The cost of 100 high-end graphics workstations at $\$ 100,000$ each would be $\$ 10,000,000$. Even if a server were to cost $\$ 2,000,000$, the cost of the server plus 100 low-cost, desktop stations at $\$ 10,000$ each would be $\$ 3,000,000$. While this cost-analysis may be over-simplified and may not be applicable to all environments, the potential for cost savings is clear.

- Pseudo-high-performance graphics to the desktop for a large community of users.

- The ability to run problems of a magnitude over and above that possible on a standalone workstation (since the equivalent of multiple cpu/memory/graphics resources is bundled together).

- Simplification of the super-high-speed communication problem between the supercomputer(s), storage, and visualization system(s) (only a few connections, and the machines can be in close proximity).

\subsection{Our Prototype Environment}

A prototype configuration which adopts the aforementioned model has been implemented at Sandia, as an adaptation of our production supercomputing environment. This configuration is depicted in Figure 3 . The configuration includes a CRAY YMP, a terabyte network storage system, and a Convex C220 which is currently acting as a visualization server. These systems are interconnected by an UltraNet, which uses an HSX interface into the CRAY, a VME interface into the network storage system, and a HiPPI into the visualization server. The visualization server has 2 cpus and 512 MB of memory, which has allowed us to experiment with some large problems. The visualization server is connected to remote desktop displays via Ethernet and FDDI. FDDI connectivity is extended from Sandia, New Mexico to Sandia's branch site in California via a long-haul FDDI/ATM interface.

FIGURE 3. Prototype Network Configuration

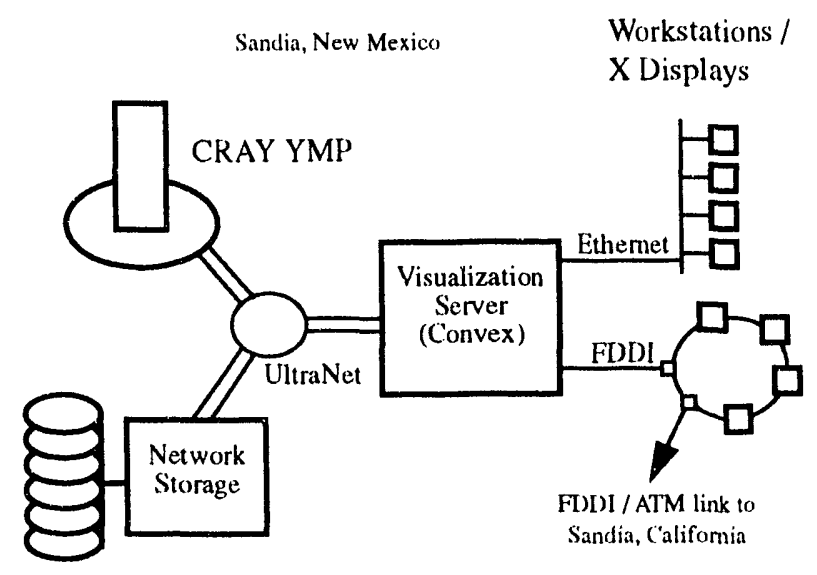


We have measured memory-to-memory data transfer rates (tep/ip) between the CRAY and the visualization server at rates up to $5 \mathrm{MB} / \mathrm{s}$. Since this is on the order of disk access rates, we can hope to access CRAY disk data from the visualization server without noticing any significant network overhead. On the other hand, NFS data rates have been slow, similar to Ethernet. It is clear that, in order to maximize record-level network-file-access data throughput. we will have to overcome the protocol limitations of NFS. FTP rates for file transfers between the CRAY, network storage, and the visualization server have been measured at about 2-3 MB/s. It is important to note that measurements were taken during normal working hours, with a normally loaded CRAY (typically saturated) and network storage system. Also, communications with the network storage system are currently bandwidth-limited by the VME interface.

The prototype visualization server is expected to be replaced in the latter part of 1993 with a new machine, which is currently being procured.

\subsection{Visualization Server Architectures}

A proposed conceptual architecture for a visualization server is shown in Figure 4.

FIGURE 4. Conceptual Visualization Server

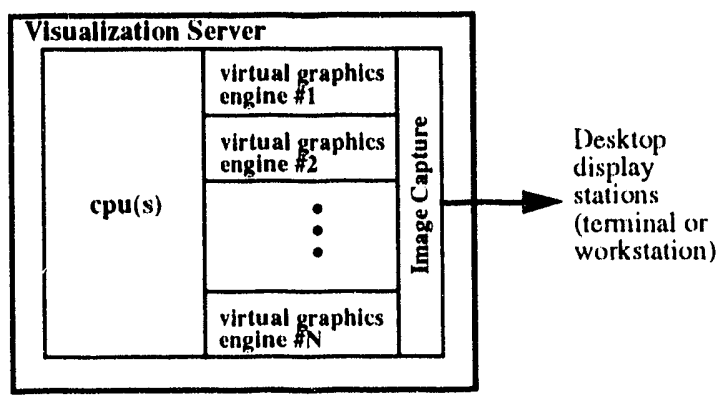

The server includes one or more processors for computation, a number of "virtual graphics engines" which support high speed rendering, and the ability to capture images produced by these virtual graphics engines and transmit the images to the desktop (for example, using $X$ Windows). The virtual graphics engines, which may be realized in the form of specialpurpose graphics hardware or as more general-purpose computational resources used to do graphics, are dynamically allocaltable to one or more visualization processes.

A more detailed characterization of the idealized requirements for a visualization server would include:

- Well-balanced computation and graphics power which can support multiple users. The architecture should be scalable to support differiag requirements based on number of simultaneous users and visualization problem complexity. 
- Configurable with lots of memory (greater than $1 \mathrm{~GB}$ ), preferably shared memory (or at least having the appearance of shared memory) which is accessible by whatever processors the system uses, at very high speed internal data rates (e.g. on the order of $100 \mathrm{MB} / \mathrm{sec}-1 \mathrm{~GB} / \mathrm{sec}$ ).

- The ability to take advantage of high-speed rendering capabilities, while delivering the resulting image into more general purpose memory, rather than to a console frame buffer.

- State-of-the-art external connectivity for the highest-speed possible communications between supercomputer(s), large storage, and the visualization server.

- Mechanisms for making best use of up-to-date, conventional communications wiring for transmission of graphical data between the visualization server and the desktop.

- A multi-user, virtual resource environment which allows complete, dynamically-allocatable access to all internal reseurces, on a demand basis. It should be possible for a large visualization task to allocate more resources (up to the whole machine, depending on availability), in order to maintain adequate response.

- Minimized contention for any physical resources which are used to realize the virtual resource environment, such as frame buffer memory and graphics pipelines.

Massively parallel (MP) processors are well suited to certain aspects of visualization, including rendering. It would seem that such processors could be used as effective components within a visualization system. However, the need to support multiple simultaneous users executing a variety of visualization processes suggests that a MIMD (Multiple Instruction Multiple Data) architecture is needed, or at least multiple SIMD (Single Instruction Multiple Data) engines which could be allocated dynamically.

A hybrid architecture which includes some combination of MP, vector, and scalar processor components, integrated with a shared memory and high-bandwidth internal communications is intriguing (see Figure 5). This architecture would allow distribution of visualization sub-processes across the various components based on which parts run best on which components. It would be particularly important for a hybrid architecture of this nature to be well-balanced in order to make effective use of each of the major processing components. 


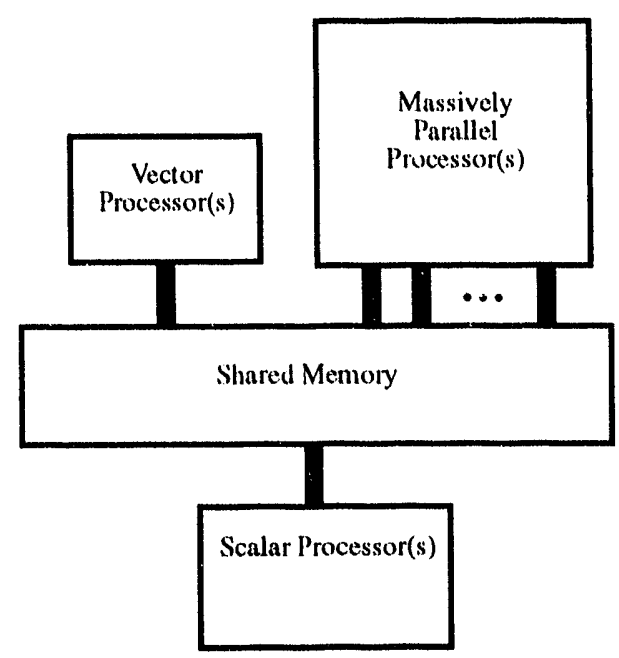

In order to understand what features make a good visualization server, it is useful to examine various existing architectures and identify strengths and weaknesses.

- Case 1. A multi-cpu, high-end graphics workstation --While this kind of system may offer adequate computational power for a modest number of users, its specialized high-speed graphics capabilities are typically only accessible from a local graphics console.

- Case 2. A traditional mini-supercomputer with a small number of processors $(<10)$-- This system offers strong computational perfornance, but lacks any specialized graphics components. All rendering is performed in software, on the general purpose cpus.

- Case 3. A traditional workstation host connected to a SIMD MP accelerator -- The MP accelerator can be used for graphics acceleration, as well as for other visualization tasks. However, the SIMD accelerator can only operate on at single user's job at any one time, resulting in contention for this resource. Multiple, perhaps smaller, MP accelerators could be used to alleviate this problem. The front end would need to be powerful enough to support multiple users, and the communications between the front end and the accelerators must be at very high speeds (on the order of $100 \mathrm{MB}$ / sec or greater).

- Case 4. A MIMD parallel machine, based on vector or superscalar cpus, with high-speed access to a pool of shared memory -- The processors can be dynamically allocated for graphics tasks and computational tasks, across multiple users. Certain processors, such as the i860, offer proven graphics performance. This architecture is attractive; however, it must be shown that such a machine can effectively deliver aggregate computational and graphics performance over its distributed architecture. 


\subsection{Getting the Picture to the Desktop}

A key to maximizing the effectiveness of a centralized visualization server is transmitting images between the visualization server and the desktop at effective rates. Nominally, we have been using conventional $X$ Windows and Ethernet to transmit images, generally requiring about $2-4$ seconds to transmit a 500x500, 8-bit image. While this performance is less than desirable, it has been acceptable in our environment -- it does not provide real-time animation, but it does provide interactive response.

- More recently, we have been very encouraged by experiments with FDDI. Using a relatively unloaded FDDI, we have been able to update images $(500 \times 5008$-bit) between the visualization server and a networked display at up to 3 frames per second (AVS running on the visualization server, rendering a simple geometry -- seə Section 6.0, Software Environment). We have also been very pleased with the recently installed long-haul FDDI/ATM link between Sandia, New Mexico, and Sandia, California [6]. In preliminary testing, usage of the visualization environment from California was virtually indistinguishable from usage of the same environment from a local networked display. When pushing images to California, we get up to about 2.5 frames per second (same test as above).

Data compression techniques may be used to further reduce the time necessary to update an image, particularly for viewing animation. In the not-too-distant future, hardware compression / decompression schemes such as JPEG and MPEG [7] should reduce the image transmission time such that reasonable quality animation (30 frames / second) will be possible over the network.

While we have focused on transmission of raster images between the visualization server and the desktop, 3-dimensional graphics protocols such as PEX provide an alternative, at least for some applications. Instead of transmitting an image, the visualization server can transmit 3D graphical data, such as polygons, to the desktop where local rendering takes place. If the local display system has sufficient power to accommodate the application's needs, this can be a very pleasing mode to work in. However, effective use in this mode requires a terminal or workstation which supports effective 3-dimensional rendering, potentially adding significant expense to the cost of a local display system.

Our preliminary experiences with PEX as a mechanism for distributing our visualization applications have not been encouraging. When using low-cost PEX systems, the performance has been inadequate for our class of problems. When using a more substantial PEX system $(\$ 25,000$ retail price, $32 \mathrm{MB}$ memory), performance was acceptable for smaller problems. However, for certain larger problems, we were unable to download the resulting 3-dimensional geometries due to insufficient memory in the local system. Finally, while PEX may be useful for generating transformed views of static 
graphical models, it is not particularly useful for viewing time-dependent animation sequences, where the graphics data changes from frame to frame, requiring downloading of now data to produce each frame.

\subsection{Software Environment}

The use of software standards, such as X11 and PEX, have and will continue to play a critical role in the success of our project. Our experiense has shown that a software environment should not be dependent on any specific computer architecture due to the transient nature of hardware. Because significant effort is required to customize software for a given application, an ideal environment would allow the replacement of the system hardware with minimal software modification.

This criteria was used in our selection of the Application Visualization System (AVS) [1] as the core of our prototype software environment. AVS satisfied our requirements because of the following features:

- Modular -- small, functional modules can be developed which can be used and reused to construct a variety of applications.

- Extensible -- functionality can be easily extended by writing application specific modules or by integrating other software tools, including commercial or public domain software.

- Distributable -- AVS applications can span across a network computing environment.

- Ubiquitous -- AVS has been ported to most workstation platforms and is not reliant on proprietary hardware.

Because AVS is a general purpose visualization toolkit, not a production system to be released to an end user, developers should anticipate spending time customizing it for their specific application. An area in which we have devoted significant effort has been database design.

At the root of our database issues is the size of results files generated on our supercomputers. Because the problems we solve are highly dynamic (nonlinear, large deformation), time-dependent solution schemes are necessary. A couple of examples which are representative of the large data sets we need to visualize are worth noting.

Figure 6 represents material boundaries from an analysis of a debris cloud consisting of 297 steel particles (red) with an average size of $.76 \mathrm{~mm}$ impacting a $12.7 \mathrm{~mm}$ aluminum target at an impact velocity of $4300 \mathrm{~m} / \mathrm{s}$. The grid size for this data was $250 \times 250 \times 100$ (6.25 million cells). Since the problem involves two materials (two fields), a total of 12.5 million data values ( 4 bytes each, $50 \mathrm{MB}$ total) were used to do the visualization. This, in turn, is only a fraction of the total memory used by the entire visualization process to produce the resulting image. This data was visualized on the Convex visu- 


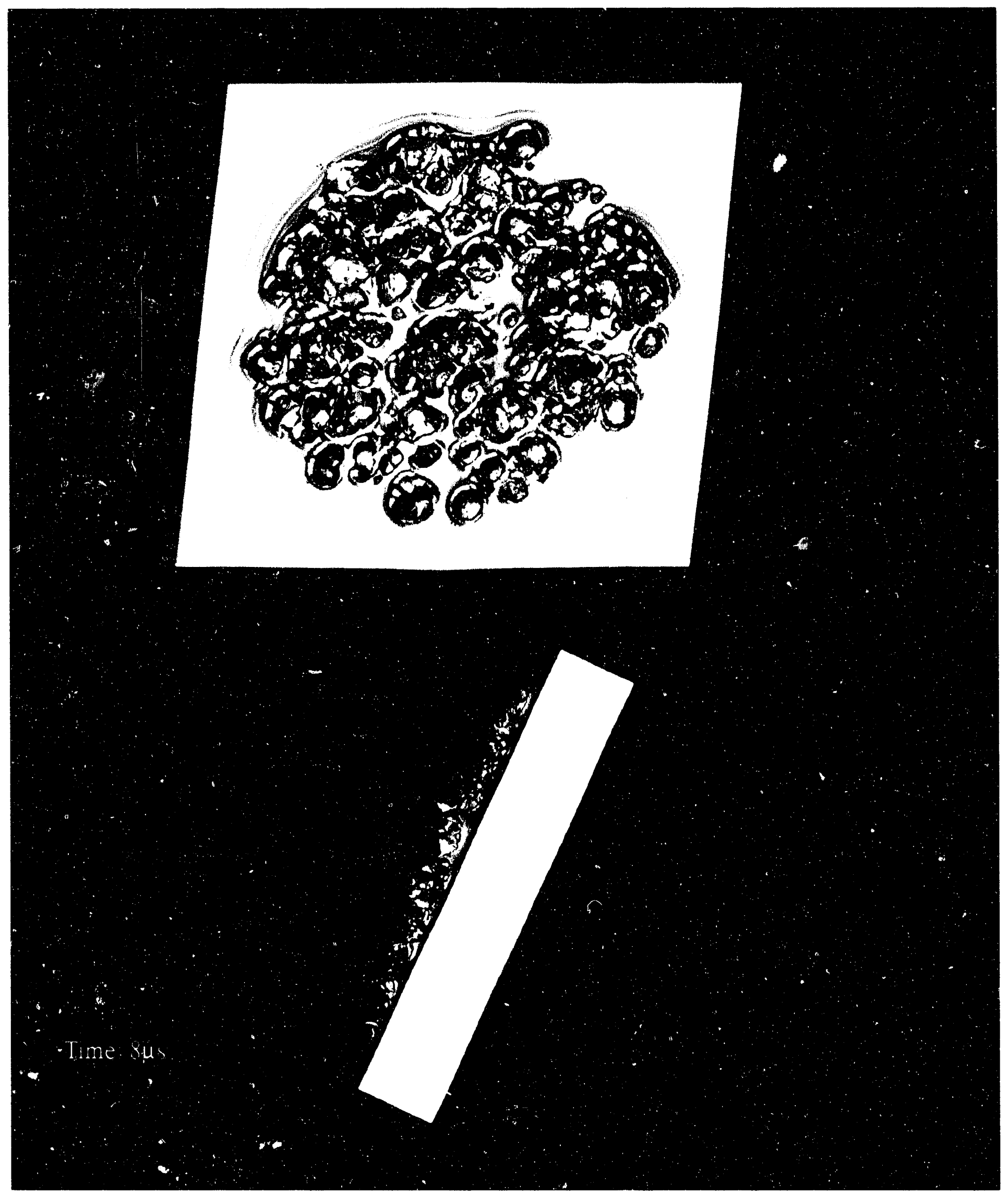

Figure 6 


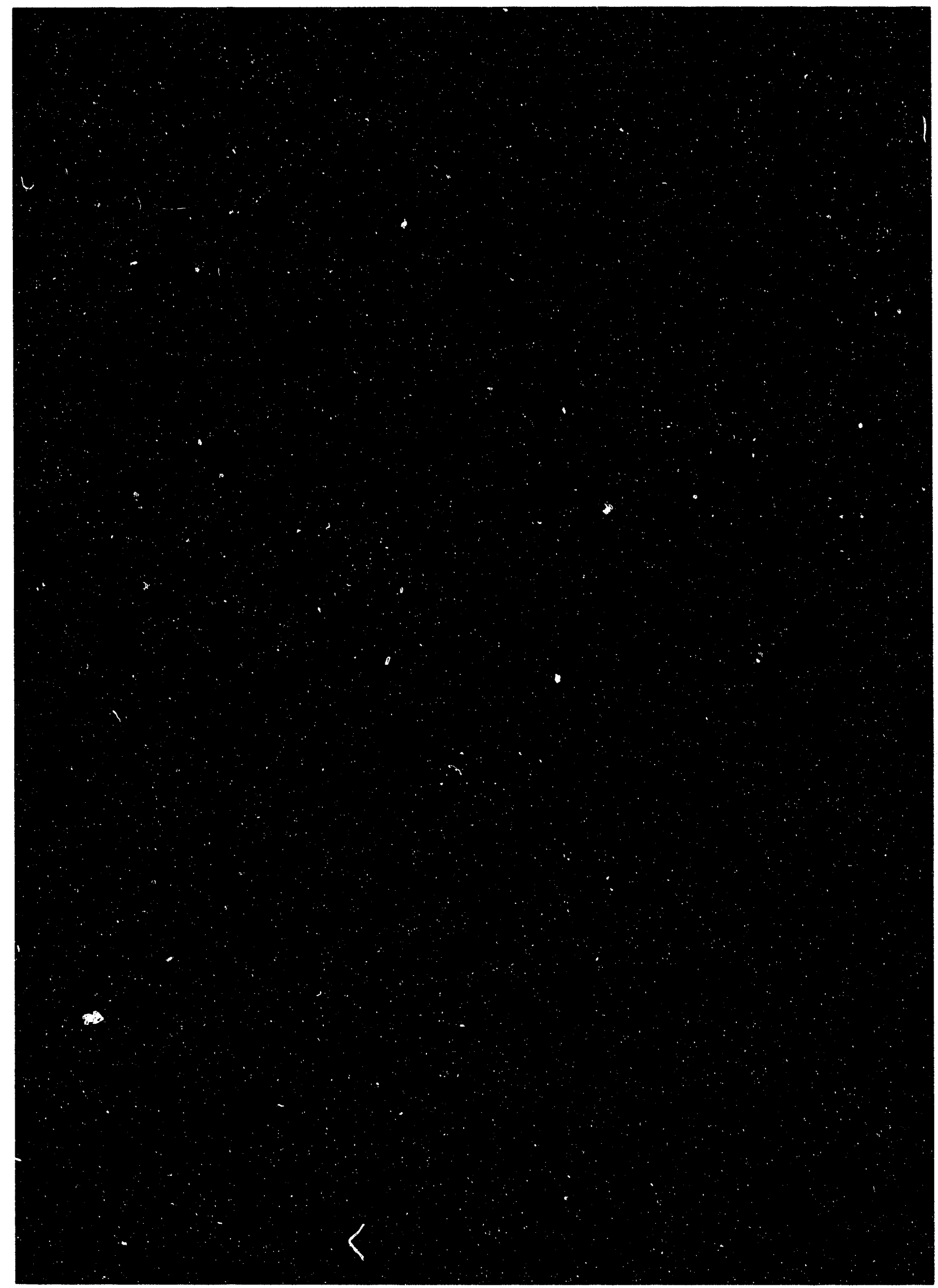


alization machine in our prototype environment, which has $512 \mathrm{MB}$ of memory -- we were unable to visualize this data on smaller conventional workstations because of insufficient memory. We were also unable to download the resulting isosurface polygons onto a PEX terminal with $32 \mathrm{MB}$ of memory, due to the number of polygons ( 3 million triangles) generated. Generating pictures of this data requires on the order of 3-5 minutes a frame on the Convex.

As an example of a large finite element data set, Figure 7 shows equivalent plastic strain on a model of a prototype joint (used to connect mechanical components) under design. The displayed model consisted of 489,000 finite elements. The analysis model was one-quarter of the displayed mu del (the entire model was not necessary due to symmetry) and was composed of 122,000 elements and 165,000 nodes. The results database (about $1.4 \mathrm{~GB}$ in size) contained over 60 time slices, with 11 variables per time slice for each element and 12 variables per time slice for each node. On our prototype visualization server, processing of a single time slice of data (including input, filtering, mapping and rendering) takes about 30 minutes. A simple geometric transformation of the object requires about 1.5 minutes (rendering only). Note that the rendering time is a small fraction of the total visualization.

Although the processing of large problems is still painful on our prototype visualization server, visualization of nominal size problems is at acceptable response levels. We expect performance to be significantly improved with the imminent purchase of a new visualization server.

Due to the criticality of the database, tasks were spawned to design or modify databases for two analysis categories: those whose geometries are structured grids and those whose geometries are unstructured grids (typically finite element analyses). After accumulating extensive input from analysts and analysis code developers, we summarized the requirements for both databases into the following functional specifications:

- C and FORTRAN library interfaces -- application codes should access the database through library routines.

- random access of data -- access any variable at any time slice.

- extensible, object-based structure -- allow the database definition to be extended for new features or data objects without necessitating the modification of all application codes that use the database.

- portable -- the database should be machine independent.

- simultaneous write/read access -- allow one application (i.e., an analysis code) to write data while another application (i.e., a visualization code) reads data; necessary for simulation tracking.

Also, the users of codes utilizing structured grids desired the following additional features.

- support for data encodings -- implement data compression techniques to reduce the size of results files. 
- allow rectilinear, cylindrical, and spherical coordinates.

Two approaches were used to address the database issues. For analyses using structured meshes, an in-house developed data file format has beer defined which allows random access as well as implementation of special compression techniques [8]. For problems solved with unstructured meshes, an application-specific interface to the netCDF [9] library, which supports random access, was created [10]. Although both of these solutions took a significant anount of time and effort to define and implement, it has become evident to us that proper data management (i.e. prudent access as well as data flow) is critical to an effective visualization process.

\subsection{Future Work}

Ultimately, we expect to install a production visualization environment for our engineering science community before the end of 1993. In the meantime, we expect to continue development and testing within our prototype environment.

A key task is to complete the acquisition of a new visualization server machine. In order to do so, we expect to benchmark a variety of potential visualization server systems. The benchmarks will be based on our specific applications, and will attempt to assess the impact of large problems as well as multiple simultaneous users. This effort should provide us with additional, concrete data regarding the effectiveness of various visualization server architectures.

Software efforts will continue to emphasize efficient processing of large, centrally-deposited visualization databases in this distributed environment. Additionally, once the high-speed integrated environment is in place, we expect to begin examining the potential use of more direct interactive visualization (versus post-processing), such as for steering our supercomputer applications.

While this work has been based in our traditional, production supercomputing environment, we are already beginning to address the integration of massively parallel machines. The impact, if any, of such machines on our distributed architecture will be assessed. The capacity of such machines to generate even greater quantities of data will further intensify our efforts to develop methods for effective processing of large problems.

\subsection{Conclusion}

In designing and prototyping an environment for highly interactive scientific visualization of large, supercomputer-generated databases, we have made the following observations: 
- The visualization process involves more than just graphics. Indeed, while well-balanced general computation and graphics power are required to insure optimal throughput, we have judged computation power to be the more critical resource. That is, given the choice of a visualization system that does superior graphics with modest computational power and another which has superior computational power with modest graphics power, the system with superior computational power would be preferred.

- A tight, high-speed integration is required between our supercomputer, large data storage facilities, and visualization components in order to allow effective processing of our large databases. Additionally, software must be implemented intelligently in order to do efficient data management.

- It is possible to implement a centralized visualization server which provides pseudo-high-performance graphics to the desktop for multiple users. The benefits of a centralized visualization server include: cost-effectiveness for a large user community; the ability to apply aggregate resources to very large problems; and simplified implementation of highspeed networking.

\section{Acknowledgments}

This work performed at Sandia National Laboratories supported by the U. S. Department of Energy under contract DEAC04-76DP00789.

Marlin Kipp and Debra Campbell for the calculations and visualization associated with Figure 6.

Gregory Sjaardema for the calculations associated with Figure 7.

\section{References}

[1] C. Upson, et al., "The Application Visualization System: A Computational Environment for Scientific Visualization," IEEE Computer Graphics and Applications, Vol. 9, No. 4, Jul. 1989.

[2] "Hardware Strategies for Scientific Visualization," Panels Proceedings SIGGRAPH '88.

[3] D. Salzman and J. Grimes, "Graphics Superworkstations and the Last Hurrah," IEEE Computer Graphics and Applications, Vol. 9, No. 4, Jul. 1989.

[4] B. Apgar, B. Bersack, and A. Mammen, "A Display System for the Stellar Graphics Supercomputer Model GS1000," Computer Graphics, ACM SIGGRAPH, Vol. 22, No. 4, Aug. 1988.

[5] R. J. Rost, J. D. Friedberg and P. L. Nishimoto, "PEX: A Network-Transparent 3D Graphics System," IEEE Computer Graphics and Applications, Jul. 1989.

[6] J. N. Naegle, N. Testi, C. Pavlakos and H. Chen, "Using a Private SMDS/ATM Network to Provide Long-Haul Supercomputing 
Visualization Services," Cray User Group Conference, Spring 1993.

[7] Communications of the ACM, Vol. 34, No. 4, Apr. 1991.

[8] C. J. Pavlakos and D. L. Camphell, "Visualization for Applications in Shock Physics," SAND 93-0916C, Sandia National Laboratories conference submittal, Mar. 1993.

[9] R. K. Rew and G. P. Davis, "NetCDF: An Interface for Scientific Data Access," IEEE Computer Graphics and Applications, Jul. 1990.

[10] L. A. Schoof, "EXODUS II: A Finite Element Data Model," SAND 92-2137, Sandia National Laboratories, in process. 

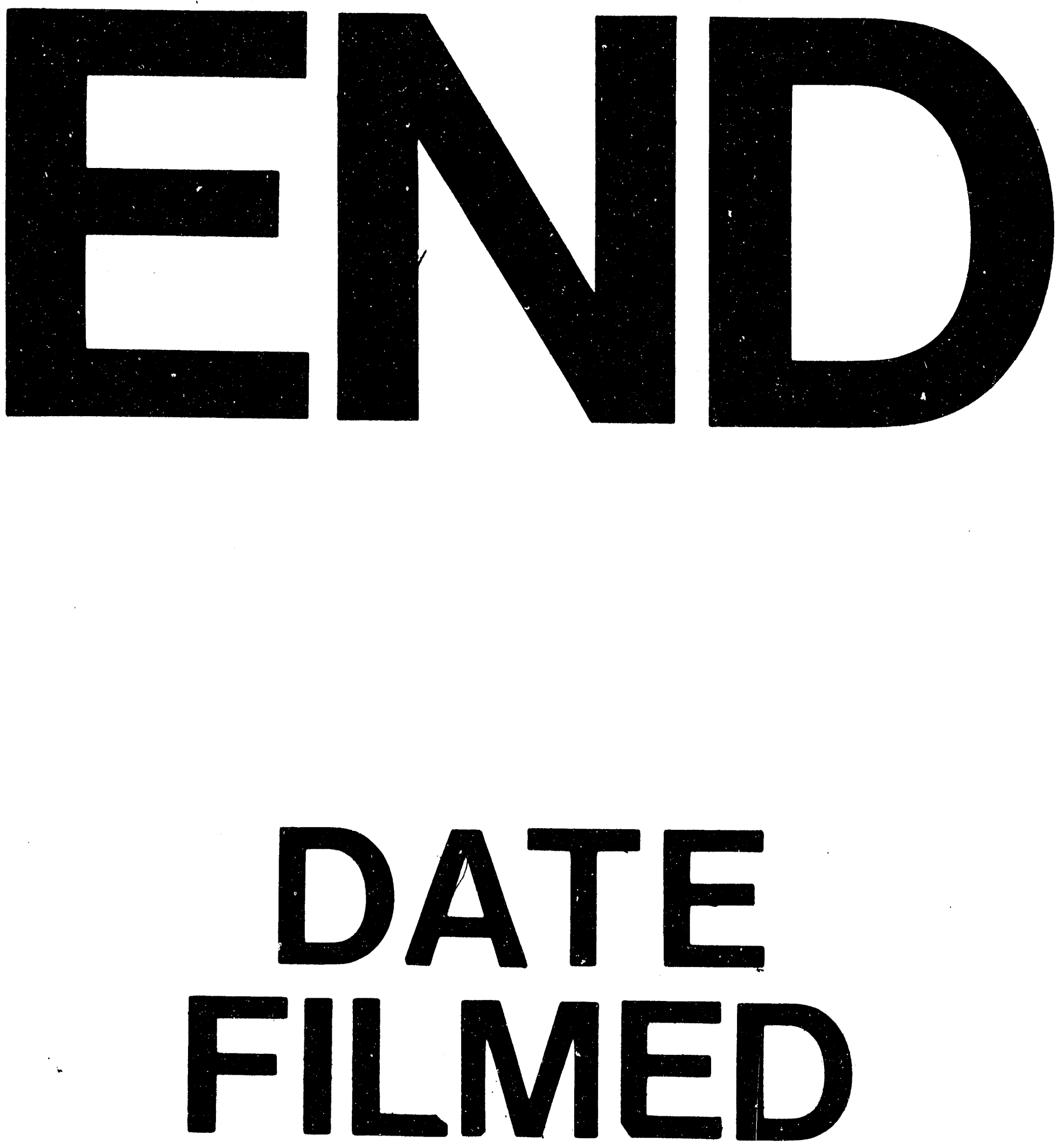

$p$

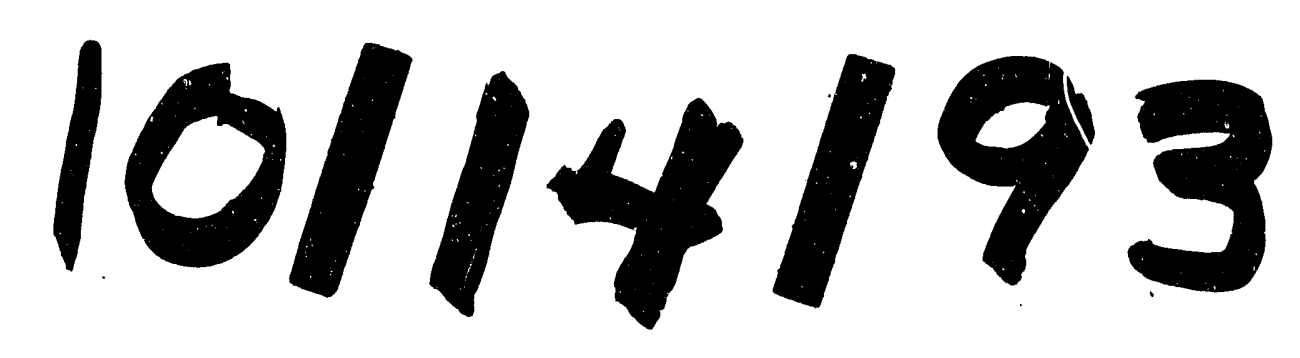


